\title{
Traditional Uses, Chemical Profile and Biological Activities of Piper hispidum Sw.: a Review
}

\author{
Wan Mohd Nuzul Hakimi Wan Salleh 1,* (D), Hakimi Kassim ${ }^{2}$ (D), Alene Tawang ${ }^{2}$ (i) \\ 1 Department of Chemistry, Faculty of Science and Mathematics, Universiti Pendidikan Sultan Idris, 35900 Tanjung Malim, \\ Perak, Malaysia; wmnhakimi@fsmt.upsi.edu.my (W.M.N.H.W.S.); \\ 2 Department of Biology, Faculty of Science and Mathematics, Universiti Pendidikan Sultan Idris, 35900 Tanjung Malim, \\ Perak, Malaysia; hakimi.kassim@fsmt.upsi.edu.my (H.K.); alene@fsmt.upsi.edu.my (A.T.); \\ * Correspondence: wmnhakimi@fsmt.upsi.edu.my (W.M.N.H.W.S)
}

Scopus Author ID 56305730900

Received: 6.01.2021; Revised: 24.01.2021; Accepted: 28.01.2021; Published: 7.02.2021

\begin{abstract}
Piper hispidum Sw. (Piperaceae) (syn. Piper hispidinervum) is a medicinal shrub distributed in Central and South America, widely used as an astringent, diuretic, stimulant for unblocking the liver and stopping hemorrhages. The plant has great interest among researchers due to the production of essential oil and important raw material for the chemical industry, which has a high demand for cosmetic, insecticide, and pesticide industries. In this review, traditional uses, phytochemicals, and biological activities of $P$. hispidum are comprehensively and systematically summarized through searching scientific databases, including Science Direct, PubMed, Google Scholar, Scopus, and Web of Science. Phytochemical studies revealed the presence of amides, benzoic acids, flavonoids, phenylpropanoids, butenolides, phenol, and essential oils; hence it has several activities, such as antioxidant, antibacterial, $\alpha$-amylase, insecticidal, schistosomicidal, leishmanial, larvicidal, antiplasmodial, cytotoxicity, estrogenic and serotonergic properties. This review is expected to draw the attention of medical professionals and the general public towards $P$. hispidum as well as to open the door for detailed research in the future.
\end{abstract}

Keywords: Piper hispidum; Piper hispidinervum; amide; benzoic acid; essential oil; leishmanial.

( 2021 by the authors. This article is an open-access article distributed under the terms and conditions of the Creative Commons Attribution (CC BY) license (https://creativecommons.org/licenses/by/4.0/).

\section{Introduction}

The Piperaceae family belongs to the Nymphaeiflorae superorder, of the Piperales order, and includes approximately 2500 species distributed in five genera (Piper, Peperomia, Zippelia, Manekia, and Verhuellia). Piperaceae can be found in herbs, vines, shrubs, and trees more rarely [1-3]. Piper is the most economically and ecologically important genus of the Piperaceae family. It is represented by herbs, shrubs, and trees and is widely distributed in the world's tropical and subtropical regions. It consists of a wide variety of species of high economic value, as they are used as food aromas, perfumes, fish venom, insecticides, as well as in the treatment of gynecological and gastrointestinal disturbances, depression, anxiety, pain, and inflammations, as well as bacterial and fungal infections [4-6]. Moreover, Piper species are used to treat diseases, including fever, jaundice, rheumatism, and neuralgia, in various countries' folk medicine [7]. Chemical studies have shown that Piper has many classes of compounds, such as amides, alkaloids, flavonoids, lignans, neolignans, aristolactams, terpenes, steroids, and phenylpropanoids [8]. 
Piper hispidum $\mathrm{Sw}$. is a herbaceous plant that grows in tropical and subtropical regions of the world, is widely distributed over a large geographic region, and can be found in Central America, the Andes, and South America. It is one of the plants that is abundantly used because of its medicinal properties [9,10]. Piper hispidinervum C.DC. is a synonym of Piper hispidum Sw., known as "pimenta-longa" in Brazil. This species resembles Piper aduncum L. to some extent but differs in its scarcely scabrous leaves, glabrous stem, and short peduncle. It is distributed throughout South America and is especially prominent in Acre's state in Brazil, and may extend into Amazonas [11].

The current review aims to provide a concise summary of the available information of $P$. hispidum (including $P$. hispidinervum), particularly concerning its traditional uses, phytochemistry, and biological activities. The pharmacological activities and chemical constituents of P. hispidum are also discussed by highlighting its pharmacological potential and identifying this plant as a natural source for potential drug compounds. This review was conducted through searches using Science Direct, PubMed, Google Scholar, Scopus, and Web of Science. The keywords used were "Piper hispidum", "Piper hispidinervum", "Piper", "phytochemistry", "biological activity", and "essential oils" articles over the period from the beginning of the database until May 2020. As a second search strategy, we included studies obtained by a manual search of the included studies' reference lists.

\section{Plant Profile}

The Plant List includes 88 synonym plant names of $P$. hispidum [12]. It is commonly known as platanillo-de-cuba and bayuyo (Cuba), cordoncillo (Mexico), jaborandi, matico or aperta-joão, and falso-jaborandi (Brazil) [13]. P. hispidum is a shrub with cylindrical and green stem, which has alternate leaves. The main anatomical characteristics that can be used in its identification are root with sclereids on cortical parenchyma, stem cortex with discontinuous strands of angular collenchyma, and vascular tissue constituted by two discontinuous circles of collateral vascular bundles. The leaf is dorsiventral and hypostomatic with tetracytic stomata. The hypodermis is discontinuous in the adaxial face, loose in the abaxial one, and presents a variable number of layers. Uniseriate epidermis and oil idioblasts occur in all organs [14].

\section{Traditional Uses}

Previous research has reported the use of $P$. hispidum to ease the pain of childbirth, anemia, and rheumatism in Nicaragua [15]. In addition, the inflorescence is applied topically for muscle aches [16]. The Q'eqchi Maya tribe in Guatemala used this plant to treat female reproductive disorders, including amenorrhea, dysmenorrhea, and menopause [17]. The plant's leaves are also traditionally used by the Chayahuitas, an Amazonian Peruvian ethnic group in Peru, to heal wounds and treat symptoms of cutaneous leishmaniasis [18]. It has also been used as an insecticide, astringent, diuretic, stimulant, liver treatment, and for stopping hemorrhages [19]. In Colombia, the tea of the decoction of P. hispidum leaves is useful for the treatment of malaria [20], while in Jamaica, the infusion of leaves in combination with $P$. aduncum is used to treat stomach pains and colds [21].

A leaf infusion is applied to kill head lice in Ecuador, while in Panama, it is used to treat conjunctivitis and diarrhea [22]. Meanwhile, the Totonacs ethnic group from Mexico used to treat mumps and tonsillitis and prevent tooth decay [23]. In South and Central America (particularly Brazil, Colombia, Ecuador, Guatemala, Honduras, Mexico, Panama, and Peru), it 
is popularly used for snakebites, insect bites, skin cleansing, head lice, amygdalitis, mouth sores, and teeth whitening agent [24]. Meanwhile, P. hispidinervum oil is an important raw source of safrole, a chemical used to synthesize piperonyl butoxide (PBO), a vital ingredient of pyrethroid insecticides [25].

\section{Phytochemistry}

Up to now, 43 compounds have been reported from $P$. hispidum including eleven amides (1-11) [26-30], seven benzoic acids (12-18) [29,31], sixteen flavonoids (19-34) [3035], five phenylpropanoids (35-39) [29,31,34], butenolides (40-42) [36], and one phenol (43) [33]. Amides are the predominant secondary metabolite constituents in P. hispidum. Their structures, names, plant part, and references are collected in Table 1 and Figure 1.

\subsection{Amides.}

Eleven amides (1-11) were successfully identified from the roots of $P$. hispidum. Recently, Lima et al. [26] reported the isolation of four amides (1-4), obtained by supercritical carbon dioxide extraction procedure. Amides (1) and (2) were found as a mixture, as determined by GC-MS. Another study, Alecio et al. [27] was successfully identified a new pyrrolidine amide, $N$-[7-(3',4'-methylenedioxyphenyl)-2(Z),4(Z)-heptadienoyl]pyrrolidine (5), along with two known amides, identified as $N$-[5-(3',4'-methylenedioxyphenyl)-2(E)pentadienoyl]-pyrrolidine (6) and $\quad N$-[2-(3',4'-methylenedioxy-6'-methoxyphenyl)-2(Z)propenoyl]-pyrrolidine (7) from the dichloromethane extract of the dried leaves part. Besides, Friedrich et al. [29] and Ruiz et al. [30] managed to isolate $N$-trans-feruloyltyramine (8) and $\mathrm{N}$-2-(3',4',5'-trimethoxyphenyl)ethyl-2-hydroxybenzamide (11) from the stems and leaves parts of $P$. hispidum, respectively. Meanwhile, Navickiene et al. [28] were successfully characterized two amides, known as (3Z,5Z)- $N$-isobutyl-8-(3',4'-methylenedioxy-phenyl)heptadienamide (9) and $\quad N$-[3-(6'-methoxy-3',4'-methylenedioxyphenyl)-2(Z)propenoyl]pyrrolidine (10).

\subsection{Benzoic acids.}

Seven benzoic acids (12-18) have been reported phytochemically from $P$. hispidum [29,31]. Friedrich et al. [29] were successfully isolated and characterized three new 4-hydroxybenzoic acid derivatives, 4-methoxy-3,5-bis-(3-hydroxy-3-methyl-1-butenyl)benzoate (12), 3hydroxy-2-(1-hydroxy-1-methylethyl)-2,3-dihydrobenzofuran-5-carboxylic acid methyl ester (13), and 3-hydroxy-2-(1-hydroxy-1-methylethyl)-2,3-dihydrobenzofuran-5-carboxylic acid (14), along with nervogenic acid (15), nervogenic acid methyl ether (16), 2,2-dimethyl-8-(3methyl-2-butenyl)-2 $H$-chromene-6-carboxylic acid (17), and 4-hydroxy-3-(3-methyl-2butenyl)benzoate (18) from the methanolic extract of the stems of $P$. hispidum.

\subsection{Flavonoids.}

Fourteen flavonoids (19-34) have been isolated phytochemically from P. hispidum [3034]. It comprises six flavanones (19-24) and nine chalcones (25-34). Erika et al. [32] were successfully characterized two known flavonoids, identified as 5-hydroxy-7methoxyflavanone (20) and 5-hydroxy-4',7-dimethoxyflavanone (21) from the ethanolic extract of the inflorescences of $P$. hispidum. In another study, Vieira et al. [33] were reported 6-hydroxy-5,7-dimethoxyflavanones (22), 8-hydroxy-5,7-dimethoxyflavanones (23), and 
5,7,8-trimethoxyflavanones (24) from the Brazillian leaves extract. In the case of chalcones, Costa et al. [35] managed to isolate three compounds, known as 2'-hydroxy-4,4',6'trimethoxychalcone (31), 2'-hydroxy-3,4,4',6'-tetramethoxychalcone (32), and 2',3-dihydroxy4,4',6'-trimethoxychalcone (33) from the same part. In the meantime, 2',4',6trimethoxychalcone (25), 2',6'-dihydroxy-4'-methoxychalcone (27), and 2'-hydroxy-3',4',6'trimethoxychalcone (28) have been isolated from the inflorescences, fruits and leaves part of P. hispidum, respectively [30-33].

Table 1. Phytochemicals identified from $P$. hispidum and bioactivities.

\begin{tabular}{|c|c|c|c|c|}
\hline No & Compounds & Part & Bioactivities & Ref \\
\hline & \multicolumn{4}{|l|}{ AMIDES } \\
\hline 1 & $\begin{array}{l}\text { (Z)-3-(6-methoxybenzo[d][1,3]dioxol-5-yl)-1- } \\
\text { (pyrrolidin-1-yl)prop-2-en-1-one }\end{array}$ & Leaves & & {$[24]$} \\
\hline 2 & $\begin{array}{l}\text { (E)-3-(6-methoxybenzo[d][1,3]dioxol-5-yl)-1- } \\
\text { (pyrrolidin-1-yl)prop-2-en-1-one }\end{array}$ & Leaves & $\begin{array}{l}\text { Cytotoxicity: Inhibition of } 9.19 \% \text { (HepG2) } \\
\text { and } 62.11 \% \text { (HL-60) }\end{array}$ & [24] \\
\hline 3 & $\begin{array}{l}\text { (2Z,4E)-7-(benzo[d][1,3]dioxol-5-yl)-1- } \\
\text { (pyrrolidin-1-yl)hepta-2,4-dien-1-one }\end{array}$ & Leaves & & {$[24]$} \\
\hline 4 & $\begin{array}{l}\text { (2E,4Z)-7-(benzo[d][1,3]dioxol-5-yl)-1- } \\
\text { (pyrrolidin-1-yl)hepta-2,4-dien-1-one }\end{array}$ & Leaves & & {$[24]$} \\
\hline 5 & $\begin{array}{l}N \text {-[7-(3',4'-methylenedioxyphenyl)-2(Z),4(Z)- } \\
\text { heptadienoyl]pyrrolidine }\end{array}$ & Leaves & $\begin{array}{l}\text { Antifungal: MIC value } 8.0 \mu \mathrm{g} \text { against } \\
\text { Cladosporium sphaerospermum }\end{array}$ & {$[25]$} \\
\hline \multirow[t]{2}{*}{6} & \multirow[t]{2}{*}{$\begin{array}{l}N \text {-[5-(3',4'-methylenedioxyphenyl)-2(E)- } \\
\text { pentadienoyl] pyrrolidine }\end{array}$} & Stems & $\begin{array}{l}\text { Antifungal: MIC value } 5.0 \mu \mathrm{g} / \mathrm{mL} \text { against } \\
\text { Cladosporium sphaerospermum }\end{array}$ & [26] \\
\hline & & Leaves & & [25] \\
\hline 7 & $\begin{array}{l}N \text {-[2-(3',4'-methylenedioxy-6'-methoxyphenyl)- } \\
\text { 2(Z)-propenoyl]-pyrrolidine }\end{array}$ & Leaves & & [25] \\
\hline 8 & $N$-trans-feruloyltyramine & Stems & & [27] \\
\hline 9 & $\begin{array}{l}(3 Z, 5 Z)-N \text {-isobutyl-8-(3',4'-methylenedioxy- } \\
\text { phenyl)-heptadienamide }\end{array}$ & Stems & $\begin{array}{l}\text { Antifungal: MIC value } 5.0 \mu \mathrm{g} / \mathrm{mL} \text { against } \\
\text { Cladosporium sphaerospermum }\end{array}$ & [26] \\
\hline 10 & $\begin{array}{l}N \text {-[3-(6'-methoxy-3',4'-methylenedioxyphenyl)- } \\
\text { 2(Z)-propenoyl]pyrrolidine }\end{array}$ & Stems & $\begin{array}{l}\text { Antifungal: MIC value } 5.0 \mu \mathrm{g} / \mathrm{mL} \text { against } \\
\text { Cladosporium sphaerospermum }\end{array}$ & {$[26]$} \\
\hline \multirow[t]{2}{*}{11} & $\begin{array}{l}N-2-\left(3^{\prime}, 4^{\prime}, 5^{\prime} \text {-trimethoxyphenyl)ethyl-2- }\right. \\
\text { hydroxybenzamide }\end{array}$ & Leaves & & [28] \\
\hline & BENZOIC ACIDS & & & \\
\hline 12 & $\begin{array}{l}\text { 4-Methoxy-3,5-bis-(3-hydroxy-3-methyl-1- } \\
\text { butenyl)benzoate }\end{array}$ & Stems & & [27] \\
\hline 13 & $\begin{array}{l}\text { 3-Hydroxy-2-(1-hydroxy-1-methylethyl)-2,3- } \\
\text { dihydrobenzofuran-5-carboxylic acid methyl } \\
\text { ester }\end{array}$ & Stems & & [27] \\
\hline 14 & $\begin{array}{l}\text { 3-Hydroxy-2-(1-hydroxy-1-methylethyl)-2,3- } \\
\text { dihydrobenzofuran-5-carboxylic acid }\end{array}$ & Stems & & [27] \\
\hline 15 & Nervogenic acid & Stems & & [27] \\
\hline \multirow[t]{2}{*}{16} & \multirow[t]{2}{*}{ Nervogenic acid methyl ether } & Stems & & [27] \\
\hline & & Fruits & & [29] \\
\hline 17 & $\begin{array}{l}\text { 2,2-Dimethyl-8-(3-methyl-2-butenyl)-2H- } \\
\text { chromene-6-carboxylic acid }\end{array}$ & Stems & & [27] \\
\hline \multirow[t]{2}{*}{18} & 4-Hydroxy-3-(3-methyl-2-butenyl)benzoate & Stems & & [27] \\
\hline & \multicolumn{4}{|l|}{ FLAVONOIDS } \\
\hline 19 & 5,7-Dihydroxyflavanone & Leaves & & [28] \\
\hline \multirow[t]{2}{*}{20} & \multirow{2}{*}{ 5-Hydroxy-7-methoxyflavanone } & Fruits & & [29] \\
\hline & & $\begin{array}{l}\text { Inflores } \\
\text { cences }\end{array}$ & & {$[30]$} \\
\hline 21 & 5-Hydroxy-4',7-dimethoxyflavanone & $\begin{array}{l}\text { Inflores } \\
\text { cences }\end{array}$ & & {$[30]$} \\
\hline 22 & 6-Hydroxy-5,7-dimethoxyflavanones & Leaves & & [31] \\
\hline 23 & 8-Hydroxy-5,7-dimethoxyflavanones & Leaves & & [31] \\
\hline 24 & 5,7,8-Trimethoxyflavanones & Leaves & & [31] \\
\hline 25 & 2',4',6-Trimethoxychalcone & $\begin{array}{l}\text { Inflores } \\
\text { cences }\end{array}$ & & [30] \\
\hline 26 & 2',4',6-Trihydroxychalcone & Leaves & & [32] \\
\hline 27 & 2',6'-Dihydroxy-4'-methoxychalcone & Fruits & & [29] \\
\hline 28 & 2'-Hydroxy-3',4',6'-trimethoxychalcone & Leaves & $\begin{array}{l}\text { Antileishmanial: } \mathrm{IC}_{50} \text { values of } 0.8 \mu \mathrm{M} \\
\text { against Leishmania amazonensis } \\
\text { Cytotoxicity: } \mathrm{IC}_{50} \text { values of } 1.6 \mu \mathrm{M} \text { against } \\
\text { peritoneal macrophages }\end{array}$ & [28] \\
\hline
\end{tabular}




\begin{tabular}{|c|c|c|c|c|}
\hline No & Compounds & Part & Bioactivities & Ref \\
\hline & & Branch & & [31] \\
\hline 29 & 2',4'-Dihydroxy-6'-methoxychalcone & Leaves & $\begin{array}{l}\text { Antileishmanial: } \mathrm{IC}_{50} \text { values of } 8.0 \mu \mathrm{M} \\
\text { against Leishmania amazonensis } \\
\text { Cytotoxicity: } \mathrm{IC}_{50} \text { values of } 18.2 \mu \mathrm{M} \text { against } \\
\text { peritoneal macrophages }\end{array}$ & [28] \\
\hline 30 & 2',3'-Dihydroxy-4',6'-dimethoxychalcones & Branch & & [31] \\
\hline 31 & 2'-Hydroxy-4,4',6'-trimethoxychalcone & Leaves & $\begin{array}{l}\text { Antimicrobial: MIC value of } 125 \text { and } 250 \\
\mu \mathrm{g} / \mathrm{mL} \text { against } S \text {. aureus and C. albicans }\end{array}$ & [33] \\
\hline 32 & 2'-Hydroxy-3,4,4',6'-tetramethoxychalcone & Leaves & $\begin{array}{l}\text { Antimicrobial: MIC value of } 250 \text { and } 500 \\
\mu \mathrm{g} / \mathrm{mL} \text { against } S \text {. aureus and C. albicans }\end{array}$ & [33] \\
\hline 33 & 2',3-Dihydroxy-4,4',6'-trimethoxychalcone & Leaves & $\begin{array}{l}\text { Antimicrobial: MIC value of } 125 \text { and } 250 \\
\mu \mathrm{g} / \mathrm{mL} \text { against } S \text {. aureus and } C \text {. albicans }\end{array}$ & [33] \\
\hline \multirow[t]{2}{*}{34} & 2',4,6'-Trihydroxy-4'-methoxychalcone & Leaves & $\begin{array}{l}\text { Antiplasmodial: } \mathrm{IC}_{50} \text { values of } 16.9 \mu \mathrm{g} / \mathrm{mL} \\
\text { (poW) and } 10.4 \mu \mathrm{g} / \mathrm{mL}(\mathrm{Dd} 2) \text { active against } \\
\text { both a chloroquine-sensitive and a resistant } \\
\text { strain of Plasmodium falciparum }\end{array}$ & [32] \\
\hline & PHENYLPROPANOIDS & & & \\
\hline 35 & $\omega$-Hydroxyisodillapiole & Stems & $\begin{array}{l}\text { Cytotoxicity: } \mathrm{IC}_{50} \text { values of } 31.8 \mu \mathrm{g} / \mathrm{mL} \\
\text { against human bladder carcinoma cell line } \\
\text { ECV-304 }\end{array}$ & [27] \\
\hline 36 & Dillapional & Stems & $\begin{array}{l}\text { Cytotoxicity: } \mathrm{IC}_{50} \text { values of } 31.7 \mu \mathrm{g} / \mathrm{mL} \\
\text { against human bladder carcinoma cell line } \\
\text { ECV-304 }\end{array}$ & [27] \\
\hline 37 & Dillapiole aldehyde & Stems & $\begin{array}{l}\text { Cytotoxicity: } \mathrm{IC}_{50} \text { values of } 31.1 \mu \mathrm{g} / \mathrm{mL} \\
\text { against human bladder carcinoma cell line } \\
\text { ECV-304 }\end{array}$ & [27] \\
\hline 38 & Dillapiole & Leaves & & [32] \\
\hline \multirow[t]{2}{*}{39} & $\begin{array}{l}\text { 1-Allyl-2,3-(methylenedioxy)-4,5-dimethoxy- } \\
\text { benzene }\end{array}$ & Fruits & & [29] \\
\hline & BUTENOLIDES & & & \\
\hline 40 & 9,10-Methylenedioxy-5,6-Z-fadyenolide & Leaves & $\begin{array}{l}\text { Estrogenic and serotonergic: Bound to the } \\
\text { serotonin receptor } 5 \text {-HT7 with IC50 values } \\
\text { of } 16.1 \text { and } 8.3 \mu \mathrm{M} \text {, respectively, and using } \\
\text { GTP shift assays, it was found to be a partial } \\
\text { agonist of the 5-HT7 receptor }\end{array}$ & [34] \\
\hline 41 & 5,6-Z-Fadyenolide & Leaves & & [34] \\
\hline \multirow[t]{2}{*}{42} & Piperolide & Leaves & & [34] \\
\hline & PHENOL & & & \\
\hline 43 & 4-(5'E-n-hexadecenyl)-phenol & Leaves & & [31] \\
\hline
\end{tabular}

\subsection{Phenylpropanoids.}

Friedrich et al. [29] managed to isolate three phenylpropanoids, identified as $\omega$ hydroxyisodillapiole (34), dillapional (35), and dillapiole aldehyde (36), from the part of the stem. Meanwhile, a long time ago, Burke and Nair, [31] reported the isolation of 1-allyl-2,3(methylenedioxy)-4,5-dimethoxy-benzene (39) from the fruits of P. hispidum.

\subsection{Butenolides.}

Three butenolides, including one new compound, 9,10-methylenedioxy-5,6-Zfadyenolide (40) were isolated from the leaves of $P$. hispidum collected from Guatemala [36]. Other butenolides were 5,6-Z-fadyenolide (41) and piperolide (42).

\subsection{Phenol.}

4-(5'E-n-hexadecenyl)-phenol (43) was the only phenol found in the leaves of $P$. hispidum, as reported by Vieira et al. [33]. 
<smiles>COc1cc2c(cc1/C=C\C(=O)N1CCCC1)OCO2</smiles><smiles>COc1cc(/C=C/C(=O)NCCc2ccc(O)cc2)ccc1O</smiles><smiles>CC(C)CNC(=O)/C=C/C=C/CCc1ccc2c(c1)OCO2</smiles><smiles>COc1cc2c(cc1/C=C/C(=O)N1CCCC1)OCO2</smiles><smiles>COc1cc(CCNC(=O)c2ccccc2O)cc(OC)c1OC</smiles>
11<smiles>CC(C)=CCc1cc(C(=O)O)cc2c1OC(C)(C)C=C2</smiles>
17<smiles>[R5]c1cc([R6])c(Br)c(C(=O)/C=C/c2ccccc2)c1[R6]</smiles>

$25 \mathrm{R}_{1}=\mathrm{R}_{3}=\mathrm{R}_{4}=\mathrm{OCH}_{3} ; \mathrm{R}_{2}=\mathrm{H}$

$26 \mathrm{R}_{1}=\mathrm{R}_{3}=\mathrm{R}_{4}=\mathrm{OH} ; \mathrm{R}_{2}=\mathrm{H}$

$\begin{array}{lll}27 & \mathrm{R}_{1}=\mathrm{R}_{4}=\mathrm{OH} ; \mathrm{R}_{3}=\mathrm{OCH}_{3} ; \mathrm{R}_{2}=\mathrm{H} & 31 \mathrm{R}_{1}=\mathrm{OH} ; \mathrm{R}_{2}=\mathrm{R}_{3}=\mathrm{R}_{4}=\mathrm{OCH}_{3} ; \mathrm{R}_{5}=\mathrm{H} \\ 28 & \mathrm{R}_{1}=\mathrm{OH} ; \mathrm{R}_{2}=\mathrm{R}_{3}=\mathrm{R}_{4}=\mathrm{OCH}_{3} & 32 \mathrm{R}_{1}=\mathrm{OH} ; \mathrm{R}_{2}=\mathrm{R}_{3}=\mathrm{R}_{4}=\mathrm{R}_{5}=\mathrm{OCH}_{3}\end{array}$

$29 \mathrm{R}_{1}=\mathrm{R}_{3}=\mathrm{OH} ; \mathrm{R}_{2}=\mathrm{H} ; \mathrm{R}_{4}=\mathrm{OC}$

$30 \mathrm{R}_{1}=\mathrm{R}_{2}=\mathrm{OH} ; \mathrm{R}_{3}=\mathrm{R}_{4}=\mathrm{OCH}_{3}$<smiles>COc1c(/C=C/C(C)(C)O)cc(C(=O)O)cc1/C=C/C(C)(C)O</smiles>

12<smiles>CCC(C)(O)[C@H]1Oc2ccc(C(=O)O)cc2[C@H]1O</smiles>
$13=\mathrm{CH}_{3}$
$14 \mathrm{R}=\mathrm{H}$<smiles>CC(C)=CCc1cc(C(=O)O)ccc1O</smiles><smiles>[R6]c1ccc(C2CC(=O)c3c(Br)cc([R6])cc3O2)cc1</smiles>

$19 \mathrm{R}_{1}=\mathrm{R}_{2}=\mathrm{OH} ; \mathrm{R}_{3}=\mathrm{H}$ $20 \mathrm{R}_{1}=\mathrm{OH} ; \mathrm{R}_{2}=\mathrm{OCH}_{3} ; \mathrm{R}_{3}=\mathrm{H}$ $21 \mathrm{R}_{1}=\mathrm{OH} ; \mathrm{R}_{2}=\mathrm{R}_{3}=\mathrm{OCH}_{3}$<smiles>[R6]c1cc([R6])c(C(=O)/C=C/c2ccc([R2])c([R5])c2)c([R])c1</smiles>

$33 \mathrm{R}_{1}=\mathrm{R}_{5}=\mathrm{OH} ; \mathrm{R}_{2}=\mathrm{R}_{3}=\mathrm{R}_{4}=\mathrm{OCH}_{3}$

$34 \mathrm{R}_{1}=\mathrm{R}_{3}=\mathrm{R}_{4}=\mathrm{OH} ; \mathrm{R}_{2}=\mathrm{OCH}_{3} ; \mathrm{R}_{5}=\mathrm{H}$<smiles>CC(C)=CCc1cc(C(=O)O)cc(CC=C(C)C)c1O</smiles>

$15 \mathrm{R}=\mathrm{H}$<smiles>[R]Cc1c([R])c2c(c(OC)c1OC)C(=O)CC(c1ccccc1)O2</smiles>

$22 \mathrm{R}_{1}=\mathrm{OH} ; \mathrm{R}_{2}=\mathrm{H}$

$23 \mathrm{R}_{1}=\mathrm{H} ; \mathrm{R}_{2}=\mathrm{OH}$

$24 \mathrm{R}_{1}=\mathrm{H} ; \mathrm{R}_{2}=\mathrm{OCH}_{3}$<smiles>[R]C=Cc1cc2c(c(OC)c1OC)OCO2</smiles>

$35 \mathrm{R}=\mathrm{CH}_{2} \mathrm{OH}$

$36 \mathrm{R}=\mathrm{CHO}$<smiles>COc1c(C=O)cc2c(c1OC)OCO2</smiles><smiles>[R2]c1c([B])c(OC)c(OC)c2c1OCO2</smiles>

$38 \mathrm{R}_{1}=\mathrm{CH}_{2} \mathrm{HC}=\mathrm{CH}_{2} ; \mathrm{R}_{2}=\mathrm{H} \quad 40 \mathrm{R}_{1}=\mathrm{R}_{2}=\mathrm{OCH}_{2} \mathrm{O}$ $39 \mathrm{R}_{1}=\mathrm{H} ; \mathrm{R}_{2}=\mathrm{CH}_{2} \mathrm{HC}=\mathrm{CH}_{2} \quad 41 \mathrm{R}_{1}=\mathrm{R}_{2}=\mathrm{H}$<smiles>[R]c1cc(/C(OC)=C2\OC(=O)C=C2OC)ccc1Br</smiles>$$
\text { (1) } \mathrm{B}_{1}=\mathrm{R}_{2}=\mathrm{H}
$$

Figure 1.

\subsection{Essential oils.}

Essential oils are complex mixtures of volatile compounds, mainly terpenes and oxygenated aromatic and aliphatic compounds, such as phenols, alcohols, aldehydes, ketones, esters, lactones, coumarins, ethers, and oxides, biosynthesized and accumulated in many plants. These naturally occurring mixtures of volatile compounds have been gaining increasing interest because of their wide range of applications in pharmaceutical, sanitary, cosmetics, perfume, food, and agricultural industries. Several significant biological activities are attributed to essential oils, such as allelopathic, antibacterial, antifungal, antioxidant, anti-inflammatory, and anticancer activities [37-48].

The chemical compositions of $P$. hispidum essential oils have been reported from various origins including Brazil [49-56], Colombia [57-59], Cuba [60], France [61], Guatemala 
[62], Panama [63], and Venezuela [64]. Most of the essential oils successfully identified monoterpenes as the major components, which are $\gamma$-terpinene, $\beta$-pinene, $\alpha$-pinene, limonene, $\delta$-3-carene, and $\alpha$-copaene. In addition, sesquiterpenes were identified as the most dominant component, which are $\beta$-caryophyllene, $\gamma$-cadinene, curzerene, and germacrene D. Besides, oxygenated sesquiterpenes characterized as trans-nerolidol, $\beta$-eudesmol, and spathulenol have been reported from $P$. hispidum essential oils collected from Colombia, Cuba, and Guatemala, respectively. $P$. hispidum essential oils also presented a phenylpropanoid, dillapiole. Its richness in the leaves and roots part was collected from Brazil and Panama. Table 2 summarizes the data on the major components and biological activities of $P$. hispidum (and $P$. hispidinervum) [65-68] essential oils.

Table 2. Components identified from $P$. hispidum and $P$. hispidinervum essential oils and bioactivities.

\begin{tabular}{|c|c|c|c|c|}
\hline \multirow[t]{2}{*}{ Country } & \multirow[t]{2}{*}{ Parts } & \multicolumn{2}{|c|}{ Total } & \multirow[t]{2}{*}{ Major components (\%) } \\
\hline & & No & $\%$ & \\
\hline Brazil & Leaves & 32 & 99.7 & $\begin{array}{l}\gamma \text {-Terpinene }(30.9 \%), \alpha \text {-terpinene } \\
(14.4 \%), p \text {-cymene }(12.1 \%), \alpha \text {-selinene } \\
(9.0 \%), \beta \text {-selinene }(8.1 \%)\end{array}$ \\
\hline Brazil & Leaves & 33 & 92.8 & $\begin{array}{l}\text { Khusimene }(12.1 \%), \beta \text {-pinene }(12.0 \%) \text {, } \\
\gamma \text {-cadinene }(13.2 \%) \text {, ledol }(8.8 \%)\end{array}$ \\
\hline Brazil & $\begin{array}{l}\text { Aerial } \\
\text { parts }\end{array}$ & 61 & 88.4 & $\begin{array}{l}\beta \text {-Caryophyllene }(10.5 \%), \alpha \text {-humulene } \\
(9.5 \%), \alpha \text {-copaene }(7.3 \%) \text {, limonene } \\
(6.9 \%) \text {, caryophyllene oxide }(5.9 \%)\end{array}$ \\
\hline
\end{tabular}

Bioactivities Ref

Antifugal: MIC and MFC [15]

values of 312.5 and $>1250$

$\mu \mathrm{g} / \mathrm{mL}$ against Rhizopus oryzae

Toxicity: $\mathrm{LC}_{50}$ value of $404.8 \mu \mathrm{g} / \mathrm{mL}$ against Artemsia salina

Cytotoxicity: $\mathrm{IC}_{50}$ value of $>25 \mu \mathrm{g} / \mathrm{mL}$ against HCT-116, SKMEL19, and ACP03 cell lines Antifungal: Detection limit (DL) of $0.1 \mu \mathrm{g} / \mathrm{mL}$ (Cladosporium cladosporioides) and 1.0 $\mu \mathrm{g} / \mathrm{mL}$ (Cladosporium sphareospermum)

Antioxidant: Inhibition of $26.4 \%$ (DPPH); TEAC (303.1 $\mathrm{mg} \mathrm{ET} / \mathrm{mL}$ ) AChE: Detection limit (DL) of $0.01 \mu \mathrm{g} / \mathrm{mL}$

\begin{tabular}{|c|c|c|c|c|c|c|}
\hline Brazil & Roots & 8 & 99.9 & $\begin{array}{l}\text { Dilapiole (57.5\%), elemicin (24.5\%), } \\
\text { apiol }(10.2 \%)\end{array}$ & & [20] \\
\hline Brazil & Leaves & 11 & 97.2 & $\begin{array}{l}\gamma \text {-Cadinene }(25.13 \%), \text { camphene } \\
(15.61 \%), \alpha \text {-guaiene }(11.47 \%), \gamma- \\
\text { elemene }(10.88 \%)\end{array}$ & & [51] \\
\hline Brazil & Leaves & 38 & 99.0 & $\begin{array}{l}\gamma \text {-Terpinene }(27.3 \%), \text { p-cymene }(14.0 \%), \\
\alpha \text {-terpinene }(12.0 \%), \alpha \text {-selinene }(8.4 \%), \\
\beta \text {-selinene }(7.5 \%), \text { terpinolene }(6.5 \%)\end{array}$ & $\begin{array}{l}\text { Antimicrobial: } \mathrm{MIC} \\
\text { value of } 937.5 \mu \mathrm{g} / \mathrm{mL} \\
\text { against Aeromonas } \\
\text { hydrophila }\end{array}$ & [52] \\
\hline Brazil & Leaves & 36 & 75.2 & $\begin{array}{l}\beta \text {-pinene }(9.76 \%), \alpha \text {-pinene }(6.90 \%),(E) \text { - } \\
\text { nerolidol }(6.30 \%), \gamma \text {-cadinene }(5.3 \%), \delta \text { - } \\
\text { cadinene }(5.0 \%)\end{array}$ & & [53] \\
\hline Brazil & Fruits & 99 & 70.0 & $\begin{array}{l}\text { limonene }(16.3 \%), \beta \text {-pinene }(14.5 \%), \alpha- \\
\text { pinene }(13.5 \%), \text { linalool }(9.6 \%), \alpha- \\
\text { terpineol }(8.5 \%), 1,8 \text {-cineol }(5.1 \%)\end{array}$ & & [54] \\
\hline \multirow[t]{2}{*}{ Brazil } & $\begin{array}{l}\text { Ripe } \\
\text { fruits }\end{array}$ & 47 & 98.1 & $\begin{array}{l}\alpha \text {-Copaene }(36.2 \%), \beta \text {-pinene }(7.5 \%), \alpha- \\
\text { pinene }(7.1 \%),(E) \text {-nerolidol }(7.0 \%)\end{array}$ & & \multirow[t]{2}{*}{ [55] } \\
\hline & $\begin{array}{l}\text { Unripe } \\
\text { fruits }\end{array}$ & 44 & 97.8 & $\begin{array}{l}\alpha \text {-copaene }(28.7 \%), \alpha \text {-pinene }(13.9 \%), \beta \text { - } \\
\text { pinene }(13.3 \%)\end{array}$ & & \\
\hline \multirow[t]{2}{*}{ Brazil } & $\begin{array}{l}\text { Fresh } \\
\text { leaves }\end{array}$ & 17 & 95.1 & $\begin{array}{l}\text { Germacrene D (33.9\%), } \delta \text {-3-carene } \\
(17.4 \%),(E) \text {-caryophyllene }(13.8 \%), \\
\text { bicyclogermacrene }(7.1 \%)\end{array}$ & & \multirow[t]{2}{*}{ [56] } \\
\hline & $\begin{array}{l}\text { dried } \\
\text { leaves }\end{array}$ & 21 & 94.1 & $\begin{array}{l}\text { Germacrene D (31.0\%), } \delta \text {-3-carene } \\
(19.1 \%),(E) \text {-caryophyllene }(14.9 \%) \text {, } \\
\text { bicyclogermacrene }(6.2 \%)\end{array}$ & $\begin{array}{l}\text { Cytotoxicity: Inhibition } \\
\text { of } 62.8 \% \text { (HepG2) and } \\
70.2 \% \text { (HL-60) } \\
\text { Larvicidal: LC }{ }_{50} \text { value of } \\
141.9 \mu \mathrm{g} / \mathrm{mL} \text { against } \\
\text { Aedes aegypti }\end{array}$ & \\
\hline
\end{tabular}




\begin{tabular}{|c|c|c|c|c|c|c|}
\hline \multirow[t]{3}{*}{ Country } & \multirow[t]{2}{*}{ Parts } & \multicolumn{2}{|c|}{ Total } & \multirow[t]{2}{*}{ Major components (\%) } & \multirow[t]{2}{*}{ Bioactivities } & \multirow[t]{3}{*}{ Ref } \\
\hline & & No & $\%$ & & & \\
\hline & Stems & 17 & 85.4 & $\begin{array}{l}\text { Germacrene D }(18.8 \%),(E) \text { - } \\
\text { caryophyllene }(14.2 \%), \delta \text {-cadinene } \\
(10.6 \%), \text { bicyclogermacrene }(9.3 \%), \alpha \text { - } \\
\text { ylangeno }(6.5 \%), \alpha \text {-muurolol }(6.2 \%)\end{array}$ & $\begin{array}{l}\text { Cytotoxicity: Inhibition } \\
\text { of } 47.7 \% \text { (HepG2) and } \\
16.3 \% \text { (HL-60) }\end{array}$ & \\
\hline Colombia & $\begin{array}{l}\text { Aerial } \\
\text { parts }\end{array}$ & 44 & 99.4 & $\begin{array}{l}\text { trans-Nerolidol }(23.6 \%), \text { caryophyllene } \\
\text { oxide }(5.4 \%), \beta \text {-elemene }(5.1 \%), \text { trans- } \beta \text { - } \\
\text { caryophyllene }(5.1 \%)\end{array}$ & $\begin{array}{l}\text { Antifungal: MIC values } \\
\text { of } 99 \mu \mathrm{g} / \mathrm{mL}(T \text {. rubrum) } \\
\text { and } 125 \mu \mathrm{g} / \mathrm{mL}(T \text {. } \\
\text { mentagrophytes) } \\
\text { Cytotoxicity: } \text { IC }_{50} \text { value } \\
\text { of } 51.7 \mu \mathrm{g} / \mathrm{mL} \text { against } \\
\text { Vero cell line }\end{array}$ & $\begin{array}{l}57] \\
{[58]}\end{array}$ \\
\hline Colombia & Leaves & 17 & 87.5 & $\begin{array}{l}\delta \text {-3-Carene }(9.6 \%), \text { p-cymene }(10.9 \%), \\
\text { limonene }(17.2 \%) \text {, elemol }(14.1), \gamma \text { - } \\
\text { elemene }(7.3 \%), \beta \text {-eudesmol }(5.7 \%)\end{array}$ & $\begin{array}{l}\text { Antifeedant: } \mathrm{EC}_{50} \text { value } \\
\text { of } 48.0 \mu \mathrm{g} / \mathrm{mL} \text { against } \\
\text { Spodoptera littoralis } \\
\text { Phytotoxic: Percentage } \\
\text { control of } 94.5 \% \text { against } \\
\text { Lolium perenne }\end{array}$ & [59] \\
\hline Cuba & Leaves & 25 & 93.5 & $\begin{array}{l}\beta \text {-eudesmol }(17.5 \%) \text {, trans-6-vinyl- } \\
4,5,6,7 \text {-tetrahydro-3,6-dimethyl-5- } \\
\text { isopropenylbenzofuran }(12.9 \%), \gamma \text { - } \\
\text { eudesmol }(9.3 \%), \alpha \text {-eudesmol }(8.1 \%) \text {, } \\
\text { elemol }(7.6 \%)\end{array}$ & & [60] \\
\hline France & Leaves & 64 & 90.5 & $\begin{array}{l}\text { Curzerene }(15.7 \%), \text { germacrene B } \\
(10.9 \%), \alpha \text {-selinene }(10.5 \%), \beta \text {-selinene } \\
(7.6 \%)\end{array}$ & $\begin{array}{l}\text { Antidermatophytic: MIC } \\
\text { value of } 62 \mu \mathrm{g} / \mathrm{mL} \text { against } \\
\text { Trichophyton } \\
\text { mentagrophytes } \\
\text { Antileishmanial: } \mathrm{IC}_{50} \\
\text { value of } 3.4 \mu \mathrm{g} / \mathrm{mL} \\
\text { against } \\
\text { Leishmania } \\
\text { amazonensis } \\
\text { Cytotoxicity: TD } 50 \text { value } \\
\text { of } 35.5 \mu \mathrm{g} / \mathrm{mL} \text { against } \\
\text { BALB/c mice peritoneal } \\
\text { macrophages }\end{array}$ & [61] \\
\hline Guatemala & Leaves & 23 & 74.9 & $\begin{array}{l}\text { Spathulenol }(8.7 \%), \beta \text {-caryophyllene } \\
(6.9 \%),(E) \text {-nerolidol }(6.8 \%) \text {, germacrene } \\
\text { D }(6.0 \%) \text {, caryophyllene oxide }(5.9 \%)\end{array}$ & & {$[62]$} \\
\hline Panama & Leaves & 43 & 95.1 & Dillapiole $(57.7 \%)$, piperitone $(10.0 \%)$ & $\begin{array}{l}\text { Larvicidal: } \mathrm{LC}_{100} \text { value } \\
\text { of } 250 \mu \mathrm{g} / \mathrm{mL} \\
\text { against Aedes aegypti }\end{array}$ & [63] \\
\hline Venezuela & Leaves & 34 & 95.2 & $\begin{array}{l}\alpha \text {-pinene }(15.3 \%), \beta \text {-pinene }(14.8 \%), \beta \text { - } \\
\text { elemene }(8.1 \%) \text {, caryophyllene oxide } \\
(7.8 \%), \delta \text {-3-carene }(6.9 \%)\end{array}$ & $\begin{array}{l}\text { Antimicrobial: } \mathrm{MBC} \\
\text { values of } 12.5 \mu \mathrm{g} / \mathrm{mL} \\
\text { against } \\
\text { S. aureus, } \text { S. epidermides, } \\
\text { S. saprophyticus, B. } \\
\text { cereus, } \text { B. subtilis } \\
\text { Cytotoxicity: IC } 50 \text { values } \\
\text { of } 18.6 \text { (HeLa), } 27.7 \\
\text { (A549), } 32.9 \text { (MCF-7), } \\
37.5 \text { (Vero) } \mu \mathrm{g} / \mathrm{mL} \\
\end{array}$ & [64] \\
\hline Brazil & Leaves & 26 & 98.6 & Safrole $(85.0 \%)$, terpinolene $(5.4 \%)$ & $\begin{array}{l}\text { Amoebicidal: At conc. of } \\
0.5 \mathrm{mg} / \mathrm{mL} \text {, the oil was } \\
\text { lethal to } 100 \% \text { of the } \\
\text { Acanthamoeba polyphaga } \\
\text { trophozoites }\end{array}$ & [65] \\
\hline Brazil & $\begin{array}{l}\text { Leaves/ } \\
\text { twigs }\end{array}$ & 24 & 98.0 & $\begin{array}{l}\text { Safrole }(77.9 \%) \text {, terpinolene }(8.8 \%) \text {, } \\
\text { bicyclogermacrene }(3.7 \%)\end{array}$ & $\begin{array}{l}\text { Antifeedant: } \mathrm{EC}_{50} \text { values } \\
\text { of } 0.4 \text { and } 17.7 \mu \mathrm{g} / \mathrm{cm}^{2} \\
\text { against } L \text {. decemlineata } \\
\text { and } S . \text { littoralis, } \\
\text { respectively }\end{array}$ & [66] \\
\hline Brazil & Leaves & 7 & 96.1 & $\begin{array}{l}\text { Safrole }(89.9 \%) \text {, terpinolene }(3.10 \%), \beta- \\
\text { bisabolene }(1.70 \%)\end{array}$ & & [67] \\
\hline Brazil & $\begin{array}{l}\text { Leaves/ } \\
\text { twigs }\end{array}$ & 22 & 99.4 & $\begin{array}{l}\text { Safrole }(64.3 \%), \text { terpinolene }(10.2 \%) \text {, } \\
(E) \text { - } \beta \text {-ocimene }(5.6 \%) \text {, }\end{array}$ & & [68] \\
\hline
\end{tabular}




\section{Biological activities}

\subsection{Antioxidant activity.}

Research by Caceres et al. [69] reported the antioxidant activity by total phenolics, DPPH, and ABTS assays. In DPPH, the methanol extract of P. hispidum showed IC50 values of $0.404 \mathrm{mg} / \mathrm{mL}$ (leaves) and $0.317 \mathrm{mg} / \mathrm{mL}$ (roots), whereas the dichloromethane extract gave IC 50 values of $0.391 \mathrm{mg} / \mathrm{mL}$ (leaves) and $0.263 \mathrm{mg} / \mathrm{mL}$ (roots), respectively. As for ABTS, the methanol extract demonstrated $\mathrm{IC}_{50}$ values of $0.498 \mathrm{mg} / \mathrm{mL}$ (leaves) and $0.131 \mathrm{mg} / \mathrm{mL}$ (roots), whereas the dichloromethane extract gave $\mathrm{IC}_{50}$ values of $0.158 \mathrm{mg} / \mathrm{mL}$ (leaves) and 0.164 $\mathrm{mg} / \mathrm{mL}$ (roots). It is also reported that dichloromethane leaves extract revealed the highest phenolic content with $14.28 \mu \mathrm{g}$ of gallic acid/mg extract.

\subsection{Antibacterial activity.}

$P$. hispidum has several medicinal properties and harbors a diversity of endophytes. Previously, four endophytic fungi from $P$. hispidum were used for obtaining crude ethyl acetate extracts that were tested against Enterococcus hirae, Escherichia coli, Micrococcus luteus, Salmonella typhi, and Staphylococcus aureus, using the cup plate technique. All bacteria were inhibited by the four extracts tested, except for Enterococcus hirae that was inhibited by only two extracts. The extract produced by $L$. theobromae was statistically the most effective against all bacteria except for $S$. typhi, being the extract of the Diaporthales endophyte more effective against it [70].

\section{3. $\alpha$-Amylase activity.}

Orlandelli et al. [71] had evaluated the suitability of four agro-industrial wastes (corncob, pineapple peel, sugarcane bagasse, and wheat bran) as low-cost substrates for the $\alpha$ amylase production by nine $P$. hispidum endophytes belonging to the genera Bipolaris, Colletotrichum, Diaporthe, Phoma, Phyllosticta, Marasmius, Phlebia, and Schizophyllum by using the starch-iodine method. Their study revealed that starchy substrates were less efficient than cellulose-rich substrates. Meanwhile, remarkable results were obtained for Bipolaris sp. JF767001 (4.14 U/mL) cultivated with pineapple peel, and for Phlebia sp. JF766997 (4.09 $\mathrm{U} / \mathrm{mL}$ ) and $S$. commune JF766994 (4.07 U/mL) with sugarcane bagasse.

\subsection{Insecticidal activity.}

Dos Santos et al. [72] evaluated the insecticidal and repellent potential of the acetonic leaf extract of $P$. hispidum on Hypothenemus hampei insects by topical application, contaminated surface, and repellent effect. They found that in the exposition in the contaminated surface, $100 \%$ of mortality was observed in the dilution of $25.0 \mathrm{mg} / \mathrm{mL}$ and 50 to $80 \%$ in dilutions of 5.0 to $0.004 \mathrm{mg} / \mathrm{mL}$, while $0.0008 \mathrm{mg} / \mathrm{mL}$ and the control resulted in only $5 \%$ of mortality. Besides, in the topic application, 60 to $65 \%$ of mortality was observed with dilutions of 25.0 to $0.1 \mathrm{mg} / \mathrm{mL}$. However, the repellence index was lower than the minimum value praised in the literature to consider a substance as a repellent.

\subsection{Schistosomicidal effects.}


The dichloromethane fraction of $P$. hispidum leaves (at $100 \mathrm{mg} / \mathrm{mL}$ ) extract was found inactive in terms of mortality, number of separated worms, and number of worms with reduced motor activity [73].

\subsection{Leishmanicidal activity.}

The ethanolic extracts of $P$. hispidum showed leishmanicidal activity against promastigotes and axenic amastigotes of Leishmania amazonensis with $\mathrm{IC}_{50}$ values of 5.0 $\mu \mathrm{g} / \mathrm{mL}$ and $69.0 \mu \mathrm{g} / \mathrm{mL}$, respectively [19].

\subsection{Larvicidal activity.}

Recently, Falkowski et al. [74] reported the leaves extract's larvicidal activity against Aedes aegypti laboratory strain susceptible to all insecticides. The extract exhibiting more than $50 \%$ larval mortality after $48 \mathrm{~h}$ of exposition at $100 \mu \mathrm{g} / \mathrm{mL}$ against the natural population were considered active. The leaves ethyl acetate extract of $P$. hispidum was found active with LC 50 values of 70.5 (95\% CI 60.4-81.6) and 54.7 (95\% CI 45.9-64.0) $\mu \mathrm{g} / \mathrm{mL}$ at 24 and $48 \mathrm{~h}$ against the laboratory strain Paea. In another study, the ethanol leaves extract of $P$. hispidum showed larvicidal potential against $A$. aegypti larvae with an $\mathrm{LC}_{50}$ value of $0.169 \mathrm{mg} / \mathrm{mL}$ [75].

\subsection{Antiplasmodial activity.}

The lipophilic extracts of $P$. hispidum proved to be active against both a chloroquinesensitive and a resistant strain of Plasmodium falciparum with IC50 values of 7.6 and 13.0 $\mu \mathrm{g} / \mathrm{mL}$, respectively [34].

\subsection{Cytotoxicity activity.}

The lipophilic extracts of $P$. hispidum reported moderately active against human tumor cell lines SK-MEL30 (melanoma) and MCF-7 (mamma cell carcinoma) with IC50 values of 24.1 and $25.1 \mu \mathrm{g} / \mathrm{mL}$, respectively. Meanwhile, the extract was found inactive against $\mathrm{KB}$ (squamous carcinoma) and A549 (lung carcinoma), which gave $\mathrm{IC}_{50}$ value $>30 \mu \mathrm{g} / \mathrm{mL}$ [34].

\subsection{Estrogenic and serotonergic activities.}

Michel et al. [36] reported the P. hispidum leaf extract enhanced the expression of the estrogen-responsive reporter and endogenous genes in MCF-7 cells, demonstrating estrogen agonist effects.

\section{Conclusions}

In conclusion, the knowledge of traditional usages, origin, phytochemicals, and biological activities on $P$. hispidum has been comprehensively studied and have been well supported and clarified by modern pharmacological studies. Thus, it is suggested the potential application of $P$. hispidum on health and pharmaceutical industry. However, the present findings are still insufficient as the study of action mechanisms is still not clearly identified. Therefore, further efforts are needed, and more well-designed studies both in vitro and in vivo are required to establish the possibilities of $P$. hispidum to be used as a food supplement or for pharmaceutical purposes. 


\section{Funding}

This research was supported by the Ministry of Education (MOE) through the Fundamental Research Grant Scheme for Research Acculturation of Early Career Researchers (FRGSRACER/1/2019/STG01/UPSI/1).

\section{Acknowledgments}

The authors would like to thank the Department of Chemistry, Faculty of Science and Mathematics, Universiti Pendidikan Sultan Idris for research facilities.

\section{Conflicts of Interest}

The authors declare no conflict of interest.

\section{References}

1. Salleh, W.M.N.H.W.; Ahmad, F.; Yen, K.H.; Sirat, H.M. Chemical compositions, antioxidant and antimicrobial activities of essential oils of Piper caninum Blume. Int. J. Mol. Sci. 2011, 12, 7720-7731, https://doi.org/10.3390/ijms12117720.

2. Salleh, W.M.N.H.W.; Ahmad, F.; Yen, K.H.; Sirat, H.M. Chemical compositions, antioxidant and antimicrobial activity of the essential oils of Piper officinarum (Piperaceae). Nat. Prod. Commun. 2012, 7 , 1934578X1200701229, https://doi.org/10.1177/1934578X1200701229.

3. Salleh, W.M.N.H.W.; Ahmad, F.; Sirat, H.M.; Yen, K.H. Chemical compositions and antibacterial activity of the leaf and stem oils of Piper porphyrophyllum (Lindl.) NE Br. EXCLI journal 2012, 11, 399, https://doi.org/10.17877/DE290R-4900.

4. Salleh, W.M.N.H.W.; Ahmad, F.; Khong, H.Y. Chemical composition of Piper stylosum Miq. and Piper ribesioides Wall. essential oils, and their antioxidant, antimicrobial and tyrosinase inhibition activities. Boletín Latinoamericano y del Caribe de Plantas Medicinales y Aromáticas 2014, 13, 488-497.

5. Salleh, W.M.N.H.W.; Ahmad, F.; Yen, K.H. Chemical compositions and antimicrobial activity of the essential oils of Piper abbreviatum, P. erecticaule and P. lanatum (Piperaceae). Nat. Prod. Commun. 2014, 9, 1934578X1400901235, https://doi.org/10.1177/1934578X1400901235.

6. Salleh, W.M.N.H.W.; Hashim, N.A.; Ahmad, F.; Heng Yen, K. Anticholinesterase and antityrosinase activities of ten Piper species from Malaysia. 2014, 4, 527-531, https://doi.org/10.5681/apb.2014.078.

7. Salleh, W.M.N.H.W.; Kamil, F.; Ahmad, F.; Sirat, H.M. Antioxidant and anti-inflammatory activities of essential oil and extracts of Piper miniatum. Nat. Prod. Commun. 2015, 10, 2005-2008, https://doi.org/10.1177/1934578X1501001151.

8. Salleh, W.; Ahmad, F.; Yen, K.H. Chemical constituents from Piper caninum and antibacterial activity. Journal of Applied Pharmaceutical Science 2015, 5, 020-025, https://doi.org/10.7324/JAPS.2015.50604.

9. Mooney, H.A.; Field, C.; Yanes, C.V.; Chu, C. Environmental controls on stomatal conductance in a shrub of the humid tropics. Proceedings of the National Academy of Sciences 1983, 80, 1295, https://doi.org/10.1073/pnas.80.5.1295.

10. Wadt, L.H.d.O.; Ehringhaus, C.; Kageyama, P.Y. Genetic diversity of "Pimenta Longa" genotypes (Piper spp., Piperaceae) of the Embrapa Acre germplasm collection. Genet. Mol. Biol. 2004, 27, 74-82, https://doi.org/10.1590/S1415-47572004000100013.

11. Yunker, T.G. Separata de hoenea: The Piperaceae of Brazil. Instituto de Botânica Press, São Paulo, SP 1972, 2, 137-139.

12. The Plant List. Version 1 2001. Available online: http://www.theplantlist.org.

13. Michel, J.L.; Duarte, R.E.; Caceres, A.; Yao, P.; Bolton, J.L.; Huang, Y.; Caceres A.; Veliz M.; Soejarto, D.D.; Mahady, G.B. Medical potential of plants used by the Q'eqchi Maya of Livingston, Guatemala for the treatment of women's health complaints. J. Ethnopharmacol. 2007, 114, 92-101.

14. Albiero, A.L.M.; Paoli, A.A.S.; Souza, L.A.; Mourao, K.S.M. Morphology and anatomy of the vegetative organs of Piper hispidum. Rev. Bras. Farmacogn. 2006, 16(3), 379-391.

15. Lans, C.; Harper, T.; Georges, K.; Bridgewater, E. Medicinal and ethnoveterinary remedies of hunters in Trinidad. BMC Complement. Altern. Med. 2001, 1, 10, https://doi.org/10.1186/1472-6882-1-10. 
16. Almeida, C.A.; Azevedo, M.; Chaves, F.; Roseo de Oliveira, M.; Rodrigues, I.A.; Bizzo, H.R.; Gama, P.E.; Alviano, D.S.; Alviano, C.S. Piper essential oils inhibit Rhizopus oryzae growth, biofilm formation, and rhizopuspepsin activity. Can. J. Infect. Dis. Med. Microbiol. 2018, 5295619, https://doi.org/10.1155/2018/5295619.

17. Coe, F.G.; Anderson, G.J. Screening of medicinal plants used by the Garífuna of Eastern Nicaragua for bioactive compounds. J. Ethnopharmacol. 1996, 53, 29-50, https://doi.org/10.1016/0378-8741(96)01424-9.

18. Duke, J.A.; Vasquez, R. Amazonian Ethnobotanical Dictionary; CRC Press: 1994.

19. Estevez, Y.; Castillo, D.; Pisango, M.T.; Arevalo, J.; Rojas, R.; Alban, J.; Deharo, E.; Bourdy, G.; Sauvain, M. Evaluation of the leishmanicidal activity of plants used by Peruvian Chayahuita ethnic group. $J$. Ethnopharmacol. 2007, 114, 254-259, https://doi.org/10.1016/j.jep.2007.08.007.

20. Morton, J.F. Atlas of medicinal plants of Middle America: Bahamas to Yucatan; Charles C. Thomas: 1981.

21. Facundo, V.A.; Pollli, A.R.; Rodrigues, R.V.; Militão, J.; Stabelli, R.G.; Cardoso, C.T. Fixed and volatile chemical constituents from stems and fruits of Piper tuberculatum Jacq. and from roots of $P$. hispidum. Acta Amazon. 2008, 38, 743-748, https://doi.org/10.1590/S0044-59672008000400018.

22. Otero, R.; Fonnegra, R.; Jiménez, S.L.; Núñez, V.; Evans, N.; Alzate, S.P.; García, M.E.; Saldarriaga, M.; Del Valle, G.; Osorio, R.G.; Díaz, A.; Valderrama, R.; Duque, A.; Vélez, H.N. Snakebites and ethnobotany in the northwest region of Colombia: Part I: Traditional use of plants. J. Ethnopharmacol. 2000, 71, 493504, https://doi.org/10.1016/s0378-8741(00)00243-9.

23. Otero, R.; Núñez, V.; Barona, J.; Fonnegra, R.; Jiménez, S.L.; Osorio, R.G.; Saldarriaga, M.; Díaz, A. Snakebites and ethnobotany in the northwest region of Colombia: Part III: Neutralization of the haemorrhagic effect of Bothrops atrox venom. J. Ethnopharmacol. 2000, 73, 233-241, https://doi.org/10.1016/s0378-8741(00)00321-4.

24. Durant-Archibold, A.A.; Santana, A.I.; Gupta, M.P. Ethnomedical uses and pharmacological activities of most prevalent species of genus Piper in Panama: A review. J. Ethnopharmacol. 2018, 217, 63-82, https://doi.org/10.1016/j.jep.2018.02.008.

25. Pimentel, F.A.; Cardoso, M.G.; Salgado, A.; Silva, V.; Zarcaroni, L.M.; Morais, A.R.; Nelson, D.L. Phytochemistry of Piper hispidinervum cultivated under the edafoclimatic conditions of Lavras, MG, Brazil. Natural Products: An Indian Journal 2006, 2, 89-94.

26. Lima, R.N.; Santos, A.D.C.; Ribeiro, A.S.; Cardozo-Filho, L.; Freitas, L.S.; Barison, A.; Costa, E.V.; Alves, P.B. Selective amides extraction and biological activity from Piper hispidum leaves using the supercritical extraction. J. Supercrit. Fluids 2020, 157, 104712, https://doi.org/10.1016/j.supflu.2019.104712.

27. Alécio, A.C.; Bolzani, V.d.S.; Young, M.C.M.; Kato, M.J.; Furlan, M. Antifungal amide from leaves of Piper hispidum. J. Nat. Prod. 1998, 61, 637-639, https://doi.org/10.1021/np9703656.

28. Navickiene, H.M.D.; Alécio, A.C.; Kato, M.J.; Bolzani, V.d.S.; Young, M.C.M.; Cavalheiro, A.J.; Furlan, M. Antifungal amides from Piper hispidum and Piper tuberculatum. Phytochemistry 2000, 55, 621-626, https://doi.org/10.1016/S0031-9422(00)00226-0.

29. Friedrich, U.; Siems, K.; Solis, P.N.; Gupta, M.P.; Jenett-Siems, K. New prenylated benzoic acid derivatives of Piper hispidum. ChemInform 2005, 36, https://doi.org/10.1002/chin.200541203.

30. Ruiz, C.; Haddad, M.; Alban, J.; Bourdy, G.; Reategui, R.; Castillo, D.; Sauvain, M.; Deharo, E.; Estevez, Y.; Arevalo, J.; Rojas, R. Activity-guided isolation of antileishmanial compounds from Piper hispidum. Phytochem. Lett. 2011, 4, 363-366, https://doi.org/10.1016/j.phytol.2011.08.001.

31. Burke, B.; Nair, M. Phenylpropene, benzoic acid and flavonoid derivatives from fruits of jamaican Piper species. Phytochemistry 1986, 25, 1427-1430, https://doi.org/10.1016/S0031-9422(00)81303-5.

32. Plazas, G.; Cuca, L.E.; Delgado, W.A. Flavonoids from inflorescences of Piper hispidum Kunth (Piperaceae) and acetylated derivatives. Rev. Colomb. Quím. 2008, 37, 135-144.

33. Vieira, P.C.; De Alvarenga, M.A.; Gottlieb, O.R.; Gottlieb, H.E. 4-Hexadecenylphenol and flavonoids from Piper hispidum. Planta Med. 1980, 39, 153-156, https://doi.org/10.1055/s-2008-1074918.

34. Jenett-Siems, K.; Mockenhaupt, F.P.; Bienzle, U.; Gupta, M.P.; Eich, E. In vitro antiplasmodial activity of Central American medicinal plants. Trop. Med. Int. Health 1999, 4, 611-615, https://doi.org/10.1046/j.13653156.1999.00456.x.

35. Costa, G.M.; Endo, E.H.; Cortez, D.A.G.; Nakamura, T.U.; Nakamura, C.V.; Dias Filho, B.P. Antimicrobial effects of Piper hispidum extract, fractions and chalcones against Candida albicans and Staphylococcus aureus. J. Mycol. Med. 2016, 26, 217-226, https://doi.org/10.1016/j.mycmed.2016.03.002.

36. Michel, J.L.; Chen, Y.; Zhang, H.; Huang, Y.; Krunic, A.; Orjala, J.; Veliz, M.; Soni, K.K.; Soejarto, D.D.; Caceres, A.; Perez, A.; Mahady, G.B. Estrogenic and serotonergic butenolides from the leaves of Piper 
hispidum Swingle (Piperaceae). J. Ethnopharmacol. 2010, 129, 220-226, https://doi.org/10.1016/j.jep.2010.03.008.

37. Salleh, W.M.N.H.W.; Khamis, S.; Nadri, M.H.; Kassim, H.; Tawang, A. Essential oil composition and antioxidant activity of Reinwardtiodendron cinereum Mabb. (Meliaceae). Nat. Volatiles Essent. Oils 2020, 7(4), 1-7, https://doi.org/10.37929/nveo.770245.

38. Salleh, W.M.N.H.W.; Khamis, S.; Nadri, M.H.; Kassim, H.; Tawang, A. Chemical composition and acetylcholinesterase inhibition of the essential oil of Cyathocalyx pruniferus (Maingay ex Hook.f. \& Thomson) J.Sinclair. Nat. Volatiles Essent. Oils 2020, 7(4), 8-13, https://doi.org/10.37929/nveo.770303.

39. Salleh, W.M.N.H.W.; Khamis, S. Chemical composition of Sarcotheca laxa (Ridl.) Knuth essential oil and their bioactivities. Riv. Ital. Sostanze Gr. 2020, 97(3), 11-16.

40. Shakri, N.M.; Salleh, W.M.N.H.W.; Khamis, S.; Ali, N.A.M. Chemical characterization of Goniothalamus macrophyllus and Goniothalamus malayanus leaves' essential oils. Z. Naturforsch. C - J Biosci. 2020, 75(1112), 485-488, https://doi.org/10.1515/znc-2020-0090.

41. Shakri, N.M.; Salleh, W.M.N.H.W.; Khamis, S.; Ali, N.A.M. Composition of the essential oils of three Malaysian Xylopia species (Annonaceae). Z. Naturforsch. C - J Biosci. 2020, 75(11-12), 479-484, https://doi.org/10.1515/znc-2020-0096.

42. Shakri, N.M.; Salleh, W.M.N.H.W.; Khamis, S.; Ali, N.A.M.; Shaharudin, S.M. Chemical composition of the essential oils of four Polyalthia species from Malaysia. Z. Naturforsch. C - J Biosci. 2020, 75(11-12), 473-478, https://doi.org/10.1515/znc-2020-0097.

43. Salleh, W.M.N.H.W.; Khamis, S. Chemical composition of the essential oil of Diospyros wallichii King \& Gamble (Ebenaceae). Nat. Volatiles Essent. Oils 2020, 7(3), 12-17.

44. Salleh, W.M.N.H.W.; Khamis, S. Chemical composition and anticholinesterase inhibitory activity of Pavetta graciliflora Wall. ex Ridl. essential oil. Z. Naturforsch. C - J Biosci. 2020, 75(11-12), 467-471, https://doi.org/10.1515/znc-2020-0075.

45. Azhar, M.A.M.; Salleh, W.M.N.H.W.; Khamis, S. Essential oil composition of three Cryptocarya species from Malaysia. Z. Naturforsch. C - J Biosci. 2020, 75(7-8), 297-301, https://doi.org/10.1515/znc-2020-0079.

46. Salleh, W.M.N.H.W.; Khamis, S.; Nafiah, M.A. Chemical composition and anticholinesterase inhibitory activity of Dipterocarpus cornutus Dyer essential oil. Z. Naturforsch. C - J Biosci. 2020, 75(5-6), 171-175, https://doi.org/10.1515/znc-2020-0028.

47. Salleh, W.M.N.H.W.; Khamis, S. Chemical composition and lipoxygenase activity of the leaves essential oil of Rothmannia macrophylla (Hook.f.) Bremek. J. Essent. Oil Bear. Pl. 2020, 23(2), 331-336, https://doi.org/10.1080/0972060X.2020.1752817.

48. Anuar, M.Z.A.; Salleh, W.M.N.H.W.; Khamis, S.; Nafiah, M.A.; Said, Z.M. Essential oil composition of Alseodaphne perakensis (Gamble) Kosterm from Malaysia. Nat. Prod. Res. 2021, 35(3), 508-511, https://doi.org/10.1080/14786419.2019.1636245.

49. Assis, A.; Brito, V.; Bittencourt, M.; Silva, L.; Oliveira, F.; Oliveira, R. Essential oils composition of four Piper species from Brazil. J. Essent. Oil Res. 2013, 25, 203-209, https://doi.org/10.1080/10412905.2013.767755.

50. da Silva, J.K.R.; Pinto, L.C.; Burbano, R.M.R.; Montenegro, R.C.; Guimarães, E.F.; Andrade, E.H.A.; Maia, J.G.S. Essential oils of Amazon Piper species and their cytotoxic, antifungal, antioxidant and anticholinesterase activities. Ind. Crops Prod. 2014, 58, 55-60, https://doi.org/10.1016/j.indcrop.2014.04.006

51. Machado, S.M.F.; Militão, J.S.L.T.; Facundo, V.A.; Ribeiro, A.; Morais, S.M.; Machado, M.I.L. Leaf oils of two brazilian Piper species: Piper arboreum Aublet var. latifolium (C.DC) Yuncker and Piper hispidum Sw. J. Essent. Oil Res. 1994, 6, 643-644, https://doi.org/10.1080/10412905.1994.9699360.

52. Majolo, C.; Monteiro, P.C.; Nascimento, A.V.P.d.; Chaves, F.C.M.; Gama, P.E.; Bizzo, H.R.; Chagas, E.C. Essential oils from five Brazilian Piper species as antimicrobials against strains of Aeromonas hydrophila. J. Essent. Oil Bear. Pl. 2019, 22, 746-761, https://doi.org/10.1080/0972060X.2019.1645047.

53. Ramos, C.S.; Soares, M.G.; da Silva, A.M.; Batista-Pereira, L.G.; Corrêa, A.G.; Kato, M.J. Electrophysiological responses of the Naupactus bipes beetle to essential oils from Piperaceae species. Nat. Prod. Commun. 2012, 7, 1934578X1200700835, https://doi.org/10.1177/1934578X1200700835.

54. Delgado, A.W.; Cuca, S.L.E. Composición química del aceite esencial de Piper hispidum. Rev. Prod. Nat. 2007, $1,5-8$.

55. Simeone, M.L.F.; Mikich, S.B.; Côcco, L.C.; Hansel, F.A.; Bianconi, G.V. Chemical composition of essential oils from ripe and unripe fruits of Piper amalago L. var. medium (Jacq.) Yunck and Piper hispidum Sw. J. Essent. Oil Res. 2011, 23, 54-58, https://doi.org/10.1080/10412905.2011.9700483. 
56. Lima, R.N.; Ribeiro, A.S.; Santiago, G.M.P.; Costa d'S.C.O.; Soares, M.B.; Bezerra, D.P.; Shanmugam, S.; dos S. Freitas, L.; Alves, P.B. Antitumor and Aedes aegypti larvicidal activities of essential oils from Piper klotzschianum, P. hispidum, and P. arboretum. Nat. Prod. Commun. 2019, 14, 1-6, https://doi.org/10.1177/1934578X19863932.

57. Benitez, N.P.; Meléndez León, E.M.; Stashenko, E.E. Essential oil composition from two species of Piperaceae family grown in Colombia. J. Chromatogr. Sci. 2009, 47, 804-807, https://doi.org/10.1093/chromsci/47.9.804.

58. Tangarife-Castaño, V.; Correa-Royero, J.B.; Roa-Linares, V.C.; Pino-Benitez, N.; Betancur-Galvis, L.A.; Durán, D.C.; Stashenko, E.E.; Mesa-Arango, A.C. Anti-dermatophyte, anti-Fusarium and cytotoxic activity of essential oils and plant extracts of Piper genus. J. Essent. Oil Res. 2014, 26, 221-227, https://doi.org/10.1080/10412905.2014.882279.

59. Jaramillo-Colorado, B.E.; Pino-Benitez, N.; González-Coloma, A. Volatile composition and biocidal (antifeedant and phytotoxic) activity of the essential oils of four Piperaceae species from Choco-Colombia. Ind. Crops Prod. 2019, 138, 111463, https://doi.org/10.1016/j.indcrop.2019.06.026.

60. Pino, J.A.; Marbot, R.; Bello, A.; Urquiola, A. Composition of the essential oil of Piper hispidum Sw. from Cuba. J. Essent. Oil Res. 2004, 16, 459-460, https://doi.org/10.1080/10412905.2004.9698771.

61. Houël, E.; Gonzalez, G.; Bessière, J.-M.; Odonne, G.; Eparvier, V.; Deharo, E.; Stien, D. Therapeutic switching: from antidermatophytic essential oils to new leishmanicidal products. Mem. Inst. Oswaldo Cruz 2015, 110, 106-113, https://doi.org/10.1590/0074-02760140332.

62. Cruz, S.M.; Cáceres, A.; Álvarez, L.E.; Apel, M.A.; Henriques, A.T. Chemical diversity of essential oils of 15 Piper species from Guatemala. 2011, 39-46, https://doi.org/10.17660/ActaHortic.2012.964.4.

63. Santana, A.I.; Vila, R.; Cañigueral, S.; Gupta, M.P. Chemical composition and biological activity of essential oils from different species of Piper from Panama. Planta Med. 2016, 82, 986-991, https://doi.org/10.1055/s0042-108060.

64. Morales, A.; Rojas, J.; Moujir, L.M.; Araujo, L.; Rondón, M. Chemical composition, antimicrobial and cytotoxic activities of Piper hispidum Sw. essential oil collected in Venezuela. J. Appl. Pharm. Sci. 2013, 3, 16, https://doi.org/10.7324/JAPS.2013.3603.

65. Sauter, I.P.; Rossa, G.E.; Lucas, A.M.; Cibulski, S.P.; Roehe, P.M.; da Silva, L.A.A.; Rott, M.B.; Vargas, R.M.F.; Cassel, E.; von Poser, G.L. Chemical composition and amoebicidal activity of Piper hispidinervum (Piperaceae) essential oil. Ind. Crops Prod. 2012, 40, 292-295, https://doi.org/10.1016/j.indcrop.2012.03.025.

66. Andrés, M.F.; Rossa, G.E.; Cassel, E.; Vargas, R.M.F.; Santana, O.; Díaz, C.E.; González-Coloma, A. Biocidal effects of Piper hispidinervum (Piperaceae) essential oil and synergism among its main components. Food Chem. Toxicol. 2017, 109, 1086-1092, https://doi.org/10.1016/j.fct.2017.04.017.

67. Riva, D.; Simionatto, E.L.; Wisniewski Jr, A.; Salerno, A.R.; Schallenberger, T.H. Adaptation studies of Piper hispidinervum C.DC.(long pepper) species in Itajaí Valley-SC, by the chemical composition of essential oil obtained by microwave and traditional hydrodistillation. Acta Amazon. 2011, 41, 297-302, https://doi.org/10.1590/S0044-59672011000200016.

68. Filho, A.P.d.S.S.; Cunha, R.L.; Vasconcelos, M.A.M.d.; Zoghbi, M.d.G.B. Essential oil components of Pogostemon heyneanus Benth, Piper hispidinervum C. DC. and Ocimum americanum L. obtained in the Amazon. J. Essent. Oil Bear. Pl. 2010, 13, 347-352, https://doi.org/10.1080/0972060X.2010.10643833.

69. Cáceres, A.; Cruz, S.M.; Gaitán, I.; Guerrero, K.; Álvarez, L.E.; Marroquín, M.N. Antioxidant activity and quantitative composition of extracts of Piper species from Guatemala with potential use in natural product industry. Acta Hortic. 2011, 77-84, https://doi.org/10.17660/ActaHortic.2012.964.9.

70. Orlandelli, R.C.; Alberto, R.N.; Almeida, T.T.; Azevedo, J.L.; Pamphile, J.A. In vitro antibacterial activity of crude extracts produced by endophytic fungi isolated from Piper hispidum Sw. J. Appl. Pharm. Sci. 2012, 2, 137-141, https://doi.org/10.7324/JAPS.2012.21027.

71. Orlandelli, R.C.; Alberto, R.N.; Almeida, T.T.; Azevedo, J.L.; Pamphile, J.A. In vitro antibacterial activity of crude extracts produced by endophytic fungi isolated from Piper hispidum Sw. J. Appl. Pharm. Sci. 2012, 2, 137-141, https://doi.org/10.4025/actascitechnol.v39i3.30067.

72. dos Santos, M.R.A.; Silva, A.G.; Lima, R.A.; Lima, D.K.S.; Sallet, L.A.P.; Teixeira, C.A.D.; Polli, A.R.; Facundo, V.A. Insecticidal activity of Piper hispidum (Piperaceae) leaves extract on (Hypothenemus hampei). Rev. Bras. Botân. 2010, 33, 319-324, https://doi.org/10.1590/S0100-84042010000200012.

73. Carrara, V.S.; Vieira, S.C.H.; De Paula, R.G.; Rodrigues, V.; Magalhães, L.G.; Cortez, D.A.G.; Da Silva Filho, A.A. In vitro schistosomicidal effects of aqueous and dichloromethane fractions from leaves and stems 
of Piper species and the isolation of an active amide from P. amalago L.(Piperaceae). J. Helminthol. 2014, 88, 321, https://doi.org/10.1017/S0022149X13000205.

74. Falkowski, M.; Jahn-Oyac, A.; Odonne, G.; Flora, C.; Estevez, Y.; Touré, S.; Boulogne, I.; Robinson, J.-C.; Béreau, D.; Petit, P.; Azam, D.; Coke, M.; Issaly, J.; Gaborit, P.; Stien, D.; Eparvier, V.; Dusfour, I.; Houël, E. Towards the optimization of botanical insecticides research: Aedes aegypti larvicidal natural products in French Guiana. Acta Trop. 2020, 201, 105179, https://doi.org/10.1016/j.actatropica.2019.105179.

75. Porto, K.R.; Motti, P.R.; Yano, M.; Roel, A.R.; Cardoso, C.A.L.; Matias, R. Screening of plant extracts and fractions on Aedes aegypti larvae found in the state of Mato Grosso do Sul (linnaeus, 1762)(culicidae). An. Acad. Bras. Cienc. 2017, 89, 895-906, https://doi.org/10.1590/0001-3765201720150017. 